\title{
Outcomes of Revision Surgery Following Instrumented Posterolateral Fusion in Degenerative Lumbar Spinal Stenosis: A Comparative Analysis between Pseudarthrosis and Adjacent Segment Disease
}

\author{
Seung-Pyo Suh ${ }^{1}$, Young-Hoon $\mathrm{Jo}^{2}$, Hae Won Jeong ${ }^{2}$, Won Rak Choi ${ }^{2}$, Chang-Nam Kang ${ }^{2}$ \\ ${ }^{1}$ Department of Orthopaedic Surgery, Sung-Ae Hospital, Seoul, Korea \\ ${ }^{2}$ Department of Orthopaedic Surgery, Hanyang University College of Medicine, Seoul, Korea
}

\section{Study Design: Retrospective study.}

Purpose: We examined the clinical and radiological outcomes of patients who received revision surgery for pseudarthrosis or adjacent segment disease (ASD) following decompression and instrumented posterolateral fusion (PLF).

Overview of Literature: At present, information regarding the outcomes of revision surgery for complications such as pseudarthrosis and ASD following instrumented PLF is limited.

Methods: This study examined 60 patients who received PLF for degenerative lumbar spinal stenosis and subsequently developed pseudarthrosis or ASD leading to revision surgery. Subjects were divided into a group of 21 patients who received revision surgery for pseudarthrosis (Group P) and a group of 39 patients who received revision surgery for ASD (Group A). Clinical outcomes were evaluated using the visual analogue scales for back pain (VAS-BP) and leg pain (VAS-LP), the Korean Oswestry disability index (K-ODI), and each patient's subjective satisfaction. Radiological outcomes were evaluated from the extent of bone union, and complications in the two groups were compared.

Results: VAS-LP at final follow-up was not statistically different between the two groups ( $p=0.353$ ), although VAS-BP and K-ODI at final follow-up were significantly worse in Group P than in Group A (all $p<0.05$ ), and only 52\% of the patients in Group P felt that their overall well-being had improved following revision surgery. Fusion rates after the first revision surgery were $71 \%(15 / 21)$ in Group $P$ and $95 \%$ (37/39) in Group A ( $p=0.018)$. The rate of reoperation was significantly higher in Group P (29\%) than in Group A (5\%) $(p=0.021)$ due to complications.

Conclusions: Clinical and radiological outcomes were worse in patients who had received revision surgery for pseudarthrosis than in those who had revision surgery for ASD. Elderly patients should be carefully advised of the risks and benefits before planning revision surgery for pseudarthrosis.

Keywords: Instrumented posterolateral fusion; Revision surgery; Pseudarthrosis; Adjacent segment disease; Outcome

Received Sep 11, 2016; Revised Nov 2, 2016; Accepted Nov 12, 2016

Corresponding author: Chang-Nam Kang

Department of Orthopaedic Surgery, Hanyang University College of Medicine,

222 Wangsimni-ro, Seongdong-gu, Seoul 04763, Korea

Tel: +82-2-2290-8485, Fax: +82-2-2299-3774, E-mail: cnkang65@hanyang.ac.kr 


\section{Introduction}

Posterolateral fusion (PLF) using pedicle screws has become a widespread technique for the surgical treatment of degenerative lumbar disease, and results in satisfactory radiological and clinical outcomes $[1,2]$. However, $9 \%-45 \%$ of such patients undergo revision surgery due to persistent pain or the recurrence of pain following initial surgery [3]. Early revision surgery is often performed because of technical errors or postoperative complications [4], whereas subsequent surgery tends to be performed because of pseudarthrosis or adjacent segment disease (ASD) $[3,5]$. Revision surgery is reported to have poorer outcomes compared to the initial surgery [6,7]. In addition, revision surgery is associated with longer operation times, greater technical challenges, and higher complication rates than primary surgery [8]. Deyo et al. [5] reported that the frequency of revision surgery for spinal stenosis decreased with increasing age and comorbidity because of the greater risks involved. As most of the patients requiring revision surgery following primary spinal fusion for degenerative lumbar spine stenosis (DLSS) are fairly old, the risks and benefits need to be carefully considered when selecting patients for this procedure [9].

The outcomes of revision surgery for complications such as pseudarthrosis and ASD following instrumented PLF are not well known, but this information is clinically important when evaluating the risks and benefits of revision surgery for specific indications, especially in the elderly. Accordingly, this study aims to evaluate and compare the clinical and radiological outcomes of patients who had received revision surgery for either pseudarthrosis or ASD following primary surgery involving decompression and instrumented PLF.

\section{Materials and Methods}

\section{Patient selection}

This study was approved by the hospital Institutional Review Board of Hanyang University College of Medicine. We identified patients who received instrumented PLF for spinal stenosis at initial surgery and selected 60 patients who received revision surgery using instrumented PLF with or without transforaminal lumbar interbody fusion (TLIF) or anterior lumbar interbody fusion (ALIF) for pseudarthrosis or ASD at the Hanyang University Medi- cal Center from January 2003 to March 2014; they were observed for a follow-up period of at least two years. At the time of the initial surgery at our hospital, patients had either grade $\mathrm{C}$ or D severe DLSS according to the criteria of Schizas et al. [10] based on magnetic resonance imaging (MRI) scans. All patients had received wide decompression and instrumented PLF for iatrogenic instability during the index surgery. At the time of revision surgery, patients had intolerable pain involving the failure of at least six months of conservative treatment or neurological deficits. Of the 60 patients, 21 received revision surgery for pseudarthrosis (Group P) and 39 for ASD (Group A), while 11 Group P patients (52\%) and 16 Group A patients (41\%) received their initial operations at other hospitals. The patient demographics of the two groups are presented in Table 1.

\section{Surgical technique}

All operations were performed by one surgeon (C.N.K.) at a single institution using a posterior approach through a midline incision in the prone position to secure full exposure of the affected area, followed by wide decompression and instrumentation using pedicle screws. During revision surgery for pseudarthrosis, TLIF with repair of PLF was performed when there was no noticeable adhesion; however, either PLF alone or ALIF with repair of PLF was performed under consideration of the age and general condition of the patients when there was noticeable adhesion. During the revision surgery for ASD, PLF with or without TLIF was performed in all cases [11]. During all fusion procedures, sufficient autogenous iliac bone grafts were used as fusion materials.

\section{Patient evaluation and follow-up}

All patients were examined before revision surgery using the visual analogue scales for back pain (VAS-BP) and leg pain (VAS-LP) and the Korean Oswestry disability index (K-ODI). Patients were asked to visit the hospital at intervals of three months for 12 months after surgery, and then six months after that for follow-up. Patients were given regular radiographic examinations and physical examinations. Until fusion was confirmed, patients were examined by computerized tomography (CT) scans at 6 months, 12 months, 18 months, and 2 years after surgery, and clinical outcomes were evaluated through a questionnaire survey 
Table 1. The comparison of the baseline data between groups

\begin{tabular}{|c|c|c|c|}
\hline Characteristic & Group $P(n=21)$ & Group A (n=39) & $p$-value \\
\hline Age (yr) & $66.1(6.7)$ & $68.2(8.3)$ & 0.100 \\
\hline Sex (male/female) & $7 / 14$ & $8 / 31$ & 0.274 \\
\hline Body mass index $\left(\mathrm{kg} / \mathrm{m}^{2}\right)$ & $27.6(4.9)$ & $26.0(4.5)$ & 0.135 \\
\hline Femoral neck BMD $\left(\mathrm{g} / \mathrm{cm}^{2}\right)$ & $0.58(0.11)$ & $0.64(0.10)$ & 0.094 \\
\hline No. of fused levels at index surgery & $3.4(1-8)$ & $2.9(1-6)$ & 0.570 \\
\hline No. of fused levels at revision surgery & $3.4(1-8)$ & $2.4(1-4)$ & 0.110 \\
\hline Length of outpatient follow-up (mo) & $37.1(24-58)$ & $37.6(24-96)$ & 0.119 \\
\hline Interval period between index surgery and revision surgery (mo) & $29.5(6-83)$ & $110.7(24-240)$ & 0.001 \\
\hline Type of revision surgery & & & 0.182 \\
\hline PLF alone (\%) & $15(71)$ & $33(85)$ & \\
\hline PLF with TLIF (\%) & $4(19)$ & $6(15)$ & \\
\hline PLF with ALIF (\%) & $2(10)$ & $0(0)$ & \\
\hline \multicolumn{4}{|l|}{ Comorbidity } \\
\hline Smoking (\%) & $3(14)$ & $1(3)$ & 0.119 \\
\hline Diabetes mellitus (\%) & $5(24)$ & $8(21)$ & 0.755 \\
\hline Rheumatoid arthritis (\%) & $6(29)$ & $3(8)$ & 0.054 \\
\hline
\end{tabular}

Values are presented as mean (standard deviation or range) or numbers (\%).

$\mathrm{BMD}$, bone mineral density; PLF, posterolateral fusion; TLIF, transforaminal lumbar interbody fusion; ALIF, anterior lumbar interbody fusion.

at an outpatient clinic or through a telephonic interview for a one month period regardless of the length of the follow-up. In this interview, we evaluated each patient's subjective satisfaction with the revision surgery, as well as VAS and K-ODI scores. Patients' subjective satisfaction was categorized as improved, unchanged, or worse.

For the radiological evaluation, two orthopedic surgeons who had not participated in the surgery (S.P.S. and Y.H.J.) measured simple radiographic and 3D-CT images taken with PACS $\pi$ view star (Infinitt, Seoul, Korea) twice at an interval of at least 2 weeks, and intra-observer and inter-observer agreements on the degree of bone union and implant failure were evaluated using kappa coefficients. Lenke fusion grades were then used to determine bone fusion after surgery [12]. Lenke A and B were considered as bone union, while Lenke $\mathrm{C}$ and $\mathrm{D}$ were considered as non-union. The final decision on bone union was based on Lenke grade and 3D-CT. Implant failure was defined as cases where the pedicle screw was pulled out or where either the pedicle screw or the metal rod was broken, and where dissociation occurred between the pedicle screw and the metal rod.

In addition to clinical and radiological outcomes, complications and reoperation rates were investigated and compared between the two groups.

\section{Statistical analysis}

PASW ver. 18.0 (SPSS Inc., Chicago, IL, USA) was used for all statistical analysis. Continuous variables were compared using the Student's $t$-test or the Mann-Whitney test, depending on whether the data were normally distributed. Paired $t$-tests or Wilcoxon signed rank tests were used to compare VAS-BP, VAS-LP, and K-ODI scores before and after surgery. The Kolmogorov-Smirov test and the Shapiro-Wilks test were used to evaluate the normality of data. The chi-square test or Fisher's exact test was used to compare dichotomous data. Differences were deemed to be statistically significant when $p<0.05$.

\section{Results}

\section{Baseline characteristics}

The two groups did not differ significantly in terms of age $(p=0.100)$, gender $(p=0.274)$, body mass index $(p=0.135)$, bone mineral density $(p=0.094)$, number of fused segments at index surgery $(p=0.570)$, number of fused seg- 
Table 2. The comparison of the clinical outcomes between groups

\begin{tabular}{|c|c|c|c|}
\hline & Group P (n=21) & Group A (n=39) & $p$-value \\
\hline \multicolumn{4}{|l|}{ VAS-BP } \\
\hline Preoperative (SD) & $7.2(1.7)$ & $7.1(1.3)$ & 0.707 \\
\hline Final follow-up (SD) & $5.0(1.6)$ & $3.8(1.6)$ & $0.006^{\text {a) }}$ \\
\hline Change (SD) & $2.2(1.5)$ & $3.2(1.5)$ & $0.047^{\mathrm{a})}$ \\
\hline \multicolumn{4}{|l|}{ VAS-LP } \\
\hline Preoperative (SD) & $5.8(2.0)$ & $6.8(2.1)$ & 0.076 \\
\hline Final follow-up (SD) & $3.1(1.7)$ & $3.5(1.8)$ & 0.353 \\
\hline Change (SD) & $2.8(1.3)$ & $3.3(1.7)$ & 0.154 \\
\hline \multicolumn{4}{|l|}{ K-ODI } \\
\hline Preoperative (SD) & $48.7(8.9)$ & $48.2(7.3)$ & 0.725 \\
\hline Final follow-up (SD) & $32.5(13.6)$ & $20.1(12.0)$ & $0.001^{a)}$ \\
\hline Change (SD) & $16.1(9.6)$ & $28.1(12.1)$ & $0.001^{a)}$ \\
\hline Patient's subjective satisfaction & & & $0.040^{a)}$ \\
\hline Improved (\%) & $11(52)$ & $32(82)$ & \\
\hline Unchanged (\%) & $4(19)$ & $4(10)$ & \\
\hline Worse (\%) & $6(29)$ & $3(8)$ & \\
\hline
\end{tabular}

Values are presented as mean (standard deviation [SD] or range) or numbers (\%).

VAS-BP, visual analog scale for back pain; VAS-LP, visual analog scale for leg pain; K-ODI, Korean Oswestry disability index.

a) Significant difference.

ments at revision surgery $(p=0.110)$, outpatient follow-up period after revision surgery $(p=0.119)$, type of surgery $(p=0.182)$, and comorbidity (all $p>0.05)$. The mean interval period between initial surgery and revision surgery was 29.5 months (range, 6-83 months) for patients in Group P and 110.7 months (range, 24-240 months) for those in Group A. The interval period was significantly longer for patients who received revision surgery for ASD $(p=0.001)$ (Table 1).

\section{Clinical outcomes}

Mean VAS-BP, VAS-LP, and K-ODI in Group P measured prior to revision surgery were $7.2,5.8$, and 48.7 , respectively, and improved to 5.0, 3.1, and 32.5 by the final follow-up (all $p=0.001$ ). Mean VAS-BP, VAS-LP, and K-ODI in Group A measured prior to revision surgery were 7.1, 6.8 , and 48.2 , respectively, and improved to $3.8,3.5$, and 20.1 by the final follow-up (all $p=0.001$ ). Thus, mean VASBP, VAS-LP, and K-ODI improved in both groups. There was no significant difference in the VAS-LP score between the two groups ( $p=0.353$ ), but the VAS-BP and K-ODI scores were significantly worse in Group P than in Group A $(p<0.05$, Table 2$)$.

At the final follow-up, only $52 \%$ of the patients in Group $P$ felt that their overall well-being had improved since the surgery, while $19 \%$ stated that their overall well-being was unchanged and 29\% felt that it had declined. In Group A, $82 \%$ of the patients felt that their overall well-being had improved since the revision surgery, while $10 \%$ felt that it was unchanged and $8 \%$ felt that it had declined (Table 2).

\section{Radiological outcomes}

The intra-observer reliability concerning the degree of bone fusion and implant failure was excellent as indicated by kappa coefficients of 0.80 (95\% confidence interval [CI], $0.68-0.92)$ and 0.85 (95\% CI, 0.72-0.98), respectively, and inter-observer reliability was good, with kappa coefficients of 0.75 (95\% CI, 0.60-0.90) and 0.76 (95\% CI, 0.66-0.86).

According to the Lenke grades determined on simple radiographs evaluated after revision surgery, Group $\mathrm{P}$ consisted of 11 cases classified as Lenke A, four as Lenke $B$, four as Lenke C, and two as Lenke D, and Group A had 32 cases classified as Lenke A, five as Lenke B, and 
Table 3. The comparison of Lenke grade between two groups

\begin{tabular}{lccc} 
Lenke grade & Group $P(n=21)$ & Group A (n=39) & $p$-value \\
A & $11(52)$ & $32(82)$ & 0.030 \\
B & $4(19)$ & $5(13)$ & \\
\hline C & $4(19)$ & $2(5)$ & \\
\hline D & $2(10)$ & 0 & \\
\hline
\end{tabular}

Values are presented as numbers (\%).

two as Lenke C (Table 3). Bone union was assessed on simple radiographic images and $3 \mathrm{D}-\mathrm{CT}$, and there were six cases of nonunion (Lenke grade C or D) in Group $\mathrm{P}$ and two (all Lenke C) in Group A. The fusion rate was higher in Group A $(95 \%, 37 / 39)$ (Fig. 1) than in Group P $(71 \%, 15 / 21)(p=0.018)$ (Fig. 2). Four of the six nonunion cases in Group P and one of the two in Group A required further secondary revision surgery as they failed to show any improvement with conservative treatment. Except for one of the cases in Group P, all secondary revision cases displayed solid union by the final follow-up. Accordingly, fusion rates at the final follow-up were $86 \%(18 / 21)$ in Group P and 97\% (38/39) in Group A ( $p=0.119)$.

There were four cases of implant failure in Group $P$ (two cases of breakage of the metal rod, two cases of screw pull-out) and two cases in Group A (due to screw pull-out) ( $p=0.171)$. All implant failures, except for one case in Group P, were related to nonunion, and except for one case of metal failure with nonunion in each group, all instances of implant failure required revision surgery (Table 4).

\section{Other complications and reoperation rates}

In addition to the cases of nonunion and implant failure, there were two cases of symptomatic ASD in each group $(p=0.606)$, but these did not require further surgery. In Group A, one patient suffered from a subjective feeling that the implant was heavy; this was removed 2.8 years after surgery. There was one case of superficial infection in each group, both of which resolved after appropriate intravenous antibiotic treatment. There was also one case of deep infection in Group P, which required surgical irrigation, debridement, and intravenous antibiotics.

In addition, one patient in Group A experienced temporary postoperative foot drop, which resolved spontane-
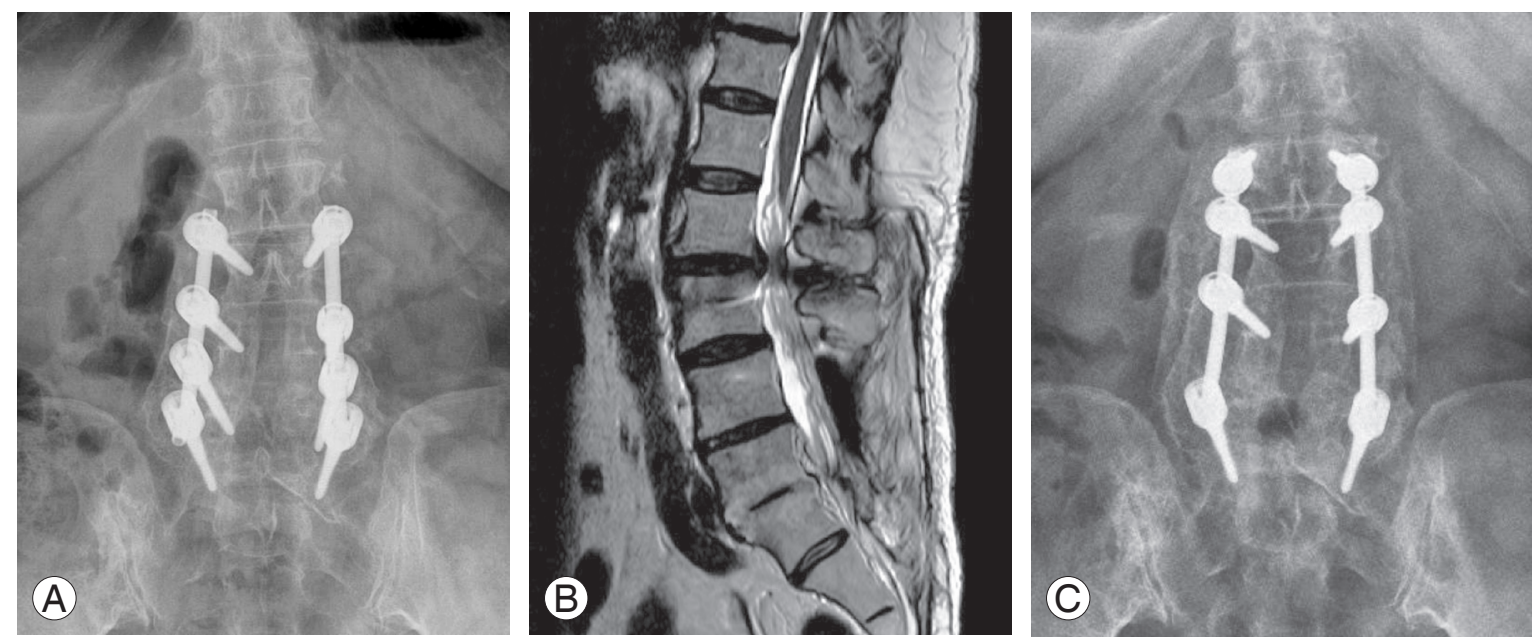

Fig. 1. (A) Simple radiograph of a 75-year-old female who received decompression and instrumented PLF from L3 to S1 and suffered ASD 10 years after surgery. (B) Before the revision surgery, severe degenerative spinal stenosis was observed by MRI at the $L 2-\mathrm{L} 3$ level. (C) A three-year follow-up simple radiograph of the same patient after an extension revision operation. Screws were removed from the fused segments (both L5 pedicle screws) and reused for the extension operation [11]. Lenke fusion grade A solid fusion was confirmed. PLF, posterolateral fusion; ASD, adjacent segment disease; MRI, magnetic resonance imaging. 

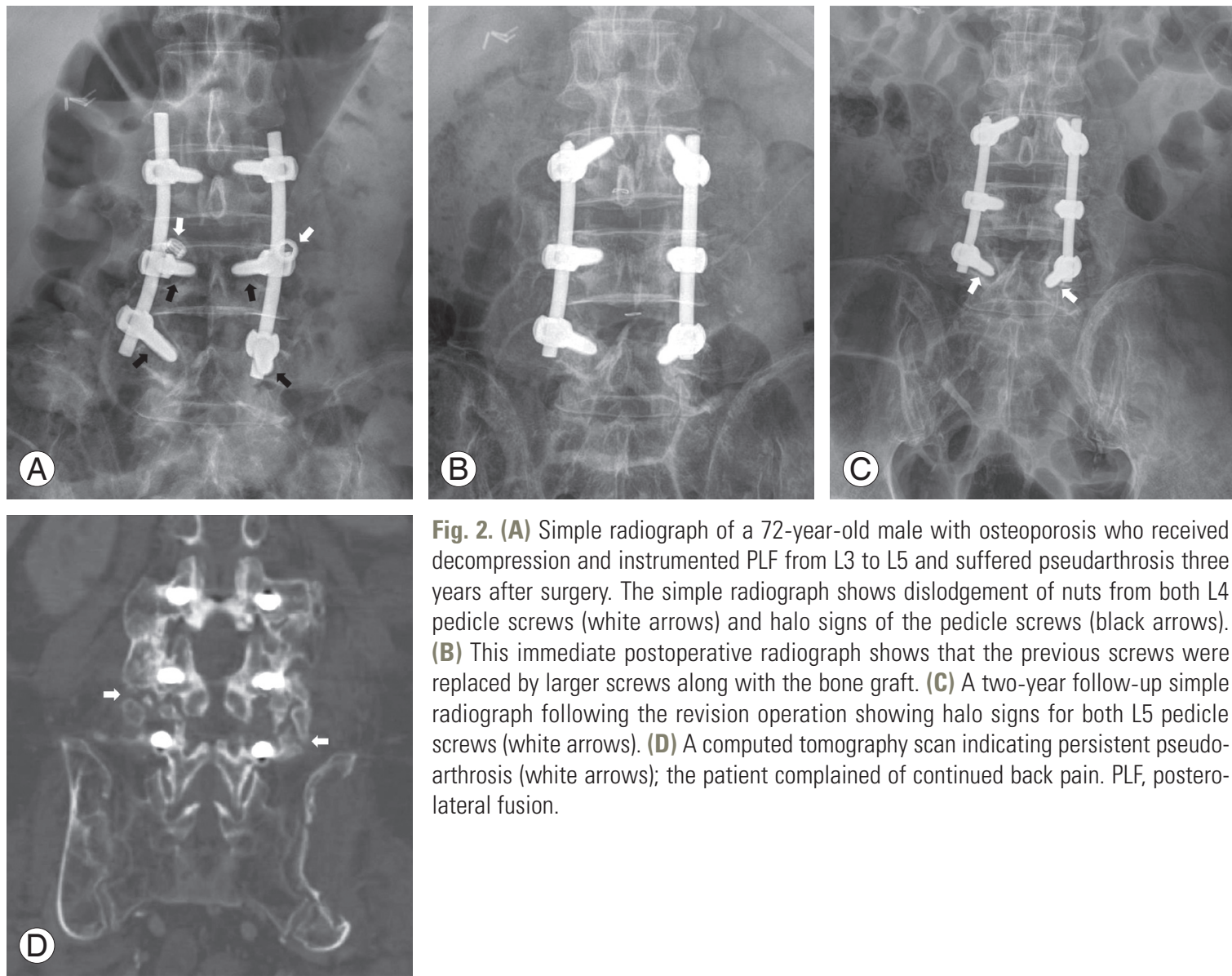

Fig. 2. (A) Simple radiograph of a 72-year-old male with osteoporosis who received decompression and instrumented PLF from $L 3$ to $L 5$ and suffered pseudarthrosis three years after surgery. The simple radiograph shows dislodgement of nuts from both $L 4$ pedicle screws (white arrows) and halo signs of the pedicle screws (black arrows).

(B) This immediate postoperative radiograph shows that the previous screws were replaced by larger screws along with the bone graft. (C) A two-year follow-up simple radiograph following the revision operation showing halo signs for both $\mathrm{L} 5$ pedicle screws (white arrows). (D) A computed tomography scan indicating persistent pseudoarthrosis (white arrows); the patient complained of continued back pain. PLF, posterolateral fusion.

ously within six months. The overall rate of complications was higher in Group P (10 patients, 48\%) than in Group A (7 patients, $18 \%)(p=0.015)$ (Table 4$)$. A total of six patients (29\%) in Group P and two (5\%) in Group A underwent additional revision procedures $(p=0.021)$ (Table 4$)$.

\section{Discussion}

In this study, patients who underwent revision surgery for pseudarthrosis following PLF for DLSS had worse clinical and radiological outcomes than those who had revision surgery for ASD. There was a significantly higher incidence of patients requiring secondary revision surgery due to nonunion after revision surgery among the former patients. The low rate of union after revision surgery probably occurred because the challenging surgical technique, which requires combined anterior and posterior approaches (leading to longer operation times and more blood loss), was avoided in elderly patients [13]. Only $29 \%$ of the patients who had revision surgery for pseudarthro- sis could undergo circumferential fusion, while the majority (71\%) only received PLF repair. PLF repair on its own for revision surgery for pseudarthrosis is complicated by a high rate of failure and persistent pseudarthrosis. West et al. [14] reported a low fusion rate (65\%) with a high incidence of unsatisfactory outcomes (47\%) in patients who received only pseudarthrosis repair using instrumented posterior arthrodesis. Lauerman et al. [7] also reported a poor fusion rate (49\%) in 40 patients who received revision surgery with instrumented PLF for pseudarthrosis. Likewise, in the present study, pseudarthrosis occurred after revision surgery in five of six (83\%) patients who received only PLF repair, although pseudarthrosis also occurred in one patient for circumferential fusion consisting of PLF and TLIF.

Pseudarthrosis has been reported to be a risk factor for persistent pseuarthrosis after revision surgery $[15,16]$, and various patient-related factors associated with this risk have been proposed. Smoking has been suggested to be a significant risk factor for pseudarthrosis in a number of 
Table 4. The comparison of complications and reoperation rate after first revision surgery

\begin{tabular}{|c|c|c|c|}
\hline & Group $P(n=21)$ & Group $A(n=39)$ & $p$-value \\
\hline \multicolumn{4}{|l|}{ Incidence of complications } \\
\hline Total & $10(48)$ & $7(18)$ & 0.015 \\
\hline Nonunion & $6(29)$ & $2(5)$ & 0.018 \\
\hline Implant failure & & & 0.171 \\
\hline Migration & $2(10)$ & $2(5)$ & \\
\hline Breakage & $2(10)$ & 0 & \\
\hline Implant heaviness & 0 & $1(3)$ & 1.000 \\
\hline Symptomatic ASD & $2(10)$ & $2(5)$ & 0.606 \\
\hline Wound infection & & & 0.275 \\
\hline Superficial & $1(5)$ & $1(3)$ & \\
\hline Deep & $1(5)$ & 0 & \\
\hline Dural tear & 0 & 0 & \\
\hline Neurologic complication & 0 & $1(3)$ & 1.000 \\
\hline \multicolumn{4}{|l|}{ Incidence of reoperations } \\
\hline Total & $6(29)$ & $2(5)$ & 0.021 \\
\hline Nonunion with implant failure & $2(10)$ & $1(3)$ & \\
\hline Nonunion without implant failure & $2(10)$ & 0 & \\
\hline Implant failure without nonunion & $1(5)$ & 0 & \\
\hline Infection & $1(5)$ & 0 & \\
\hline Implant heaviness & 0 & $1(3)$ & \\
\hline
\end{tabular}

Values are presented as numbers (\%).

ASD, adjacent segment disease.

studies [15], and patients with rheumatoid arthritis (RA) often have poor bone quality, which offers weak fixation strength for pedicle screws, thus leading to pseudarthrosis [17-19]. In fact, of our six patients with persistent pseudarthrosis, two were heavy smokers and three had RA with osteoporosis. Accordingly, such persistent patient-related factors may have been responsible for the poorer fusion rate in patients who had revision surgery for pseuarthrosis compared to those who had revision surgery for ASD. Although not statistically significant given the low numbers of patients in this study, there was a strong trend toward a higher incidence of RA and lower BMD scores in Group P compared to Group A.

The final fusion rate was comparable in the two groups, although there was a significantly higher incidence of secondary revision surgery in Group P. However, clinical outcomes in terms of VAS-BP, K-ODI score, and the patient's subjective satisfaction were better in Group A. Jonsson and Stromqvis [20] reported that multiple revision operations had inferior outcomes. Increased epidural scarification was found to be unavoidable in patients with multiple revisions [21], and there is a significant association between extensive epidural scarring and poor clinical outcomes [22].

The interval between the index and revision surgery may also affect clinical outcomes. In this study, the mean interval between index surgery and revision surgery was 9.2 years in Group A and 2.5 years in Group P. This interval for ASD has been reported to range from 4.4 to 11.5 years $[6,23,24]$. Furthermore, the length of the pain-free interval has been shown to be positively correlated with successful outcomes of revision surgery $[16,20,25]$. Jonsson and Stromqvis [20] reported degenerative condition as a factor for pain in patients with significant pain-free intervals and superior clinical outcomes when revision was performed for such degenerative causes.

One of the few limitations of this study is that it was performed retrospectively and that an insufficient number of patients receiving revision surgery were enrolled. The reason for the revision surgery group being small was 
that the study was limited to patients who had previously received decompression and instrumented PLF for DLSS. Another limitation is that the posterior approach on its own was selected for most of the patients with pseudarthrosis. It is possible that a higher fusion rate would have been achieved by performing operations using the combined anterior and posterior approach; however, the resulting longer operation time and increased blood loss might have caused additional complications in elderly patients [9].

\section{Conclusions}

We conclude that patients who underwent revision surgery for pseudarthrosis had worse clinical and radiological outcomes than those who underwent revision surgery for ASD. Elderly patients should be carefully counseled about the risks and benefits of this procedure before planning revision surgery for pseuarthrosis.

\section{Conflict of Interest}

No potential conflict of interest relevant to this article was reported.

\section{ORCID}

Seung-Pyo Suh: 0000-0002-1457-8984

Young-Hoon Jo: 0000-0002-4299-2496

Hae Won Jeong: 0000-0001-9599-907X

Won Rak Choi: 0000-0002-0854-7567

Chang-Nam Kang: 0000-0002-4633-3391

\section{References}

1. Turunen V, Nyyssonen T, Miettinen H, et al. Lumbar instrumented posterolateral fusion in spondylolisthetic and failed back patients: a long-term followup study spanning 11-13 years. Eur Spine J 2012;21: 2140-8.

2. Andersen T, Videbaek TS, Hansen ES, Bunger C, Christensen FB. The positive effect of posterolateral lumbar spinal fusion is preserved at long-term follow-up: a RCT with 11-13 year follow-up. Eur Spine J 2008;17:272-80.

3. Kelly MP, Lenke LG, Bridwell KH, Agarwal R, Godzik J, Koester L. Fate of the adult revision spinal deformity patient: a single institution experience. Spine (Phila Pa 1976) 2013;38:E1196-200.

4. Hazard RG. Failed back surgery syndrome: surgical and nonsurgical approaches. Clin Orthop Relat Res 2006;443:228-32.

5. Deyo RA, Martin BI, Kreuter W, Jarvik JG, Angier H, Mirza SK. Revision surgery following operations for lumbar stenosis. J Bone Joint Surg Am 2011;93:197986.

6. Djurasovic M, Glassman SD, Howard JM, Copay AG, Carreon LY. Health-related quality of life improvements in patients undergoing lumbar spinal fusion as a revision surgery. Spine (Phila Pa 1976) 2011;36:26976.

7. Lauerman WC, Bradford DS, Ogilvie JW, Transfeldt EE. Results of lumbar pseudarthrosis repair. J Spinal Disord 1992;5:149-57.

8. Galhom AE, Elhadi M. Revision surgery for failed back: techniques, outcome, and complications. Med J Cairo Univ 2013;81:115-22.

9. Carreon LY, Puno RM, Dimar JR 2nd, Glassman SD, Johnson JR. Perioperative complications of posterior lumbar decompression and arthrodesis in older adults. J Bone Joint Surg Am 2003;85:2089-92.

10. Schizas C, Theumann N, Burn A, et al. Qualitative grading of severity of lumbar spinal stenosis based on the morphology of the dural sac on magnetic resonance images. Spine (Phila Pa 1976) 2010;35:191924.

11. Lee JK, Jo YH, Kang CN. Cost-effectiveness analysis of existing pedicle screws reusing technique in extension revision operation for adjacent segmental stenosis after lumbar posterolateral fusion. Spine (Phila $\mathrm{Pa}$ 1976) 2016;41:E785-90.

12. Lenke LG, Bridwell KH, Bullis D, Betz RR, Baldus C, Schoenecker PL. Results of in situ fusion for isthmic spondylolisthesis. J Spinal Disord 1992;5:433-42.

13. Han X, Zhu Y, Cui C, Wu Y. A meta-analysis of circumferential fusion versus instrumented posterolateral fusion in the lumbar spine. Spine (Phila Pa 1976) 2009;34:E618-25.

14. West JL 3rd, Bradford DS, Ogilvie JW. Results of spinal arthrodesis with pedicle screw-plate fixation. J Bone Joint Surg Am 1991;73:1179-84.

15. Gertzbein SD, Hollopeter MR, Hall S. Pseudarthrosis of the lumbar spine. Outcome after circumferential fusion. Spine (Phila Pa 1976) 1998;23:2352-6. 
16. Lehmann TR, LaRocca HS. Repeat lumbar surgery. A review of patients with failure from previous lumbar surgery treated by spinal canal exploration and lumbar spinal fusion. Spine (Phila Pa 1976) 1981;6:615-9.

17. Yamagata M, Kitahara H, Minami S, et al. Mechanical stability of the pedicle screw fixation systems for the lumbar spine. Spine (Phila Pa 1976) 1992;17:S51-4.

18. Kumano K, Hirabayashi S, Ogawa Y, Aota Y. Pedicle screws and bone mineral density. Spine (Phila Pa 1976) 1994;19:1157-61.

19. Kang CN, Kim CW, Moon JK. The outcomes of instrumented posterolateral lumbar fusion in patients with rheumatoid arthritis. Bone Joint J 2016;98B: 102-8.

20. Jonsson B, Stromqvist B. Repeat decompression of lumbar nerve roots: a prospective two-year evalua- tion. J Bone Joint Surg Br 1993;75:894-7.

21. Fritsch EW, Heisel J, Rupp S. The failed back surgery syndrome: reasons, intraoperative findings, and longterm results: a report of 182 operative treatments. Spine (Phila Pa 1976) 1996;21:626-33.

22. Robertson JT. Role of peridural fibrosis in the failed back: a review. Eur Spine J 1996;5 Suppl 1:S2-6.

23. Whitecloud TS 3rd, Davis JM, Olive PM. Operative treatment of the degenerated segment adjacent to a lumbar fusion. Spine (Phila Pa 1976) 1994;19:531-6.

24. Lee CS, Hwang CJ, Lee SW, et al. Risk factors for adjacent segment disease after lumbar fusion. Eur Spine J 2009;18:1637-43.

25. Stewart G, Sachs BL. Patient outcomes after reoperation on the lumbar spine. J Bone Joint Surg Am 1996; 78:706-11. 